\title{
Crossed Cerebrocerebellar Language Lateralization: An Additional Diagnostic Feature for Assessing Atypical Language Representation in Presurgical Functional MR Imaging
}

\author{
C. Méndez Orellana, E. Visch-Brink, M. Vernooij, S. Kalloe, D. Satoer, A. Vincent, A. van der Lugt, and M. Smits
}

\begin{abstract}
BACKGROUND AND PURPOSE: Determining language dominance with $\mathrm{fMRI}$ is challenging in patients with brain tumor, particularly in cases of suspected atypical language representation. Supratentorial activation patterns must be interpreted with great care when the tumor is in or near the presumed language areas, where tumor tissue or mass effect can lead to false-negative fMRI results. In this study, we assessed cerebrocerebellar language fMRI lateralization in healthy participants and in patients with brain tumors with a focus on atypical language representation.
\end{abstract}

MATERIALS AND METHODS: Twenty healthy participants and 38 patients with a brain tumor underwent fMRI with a verb-generation task. Cerebral and cerebellar language lateralizations were separately classified as left-sided, right-sided, or symmetric. Electrocortical stimulation was performed in 19 patients. With the McNemar test, we evaluated the dependency between language lateralization in the cerebrum and cerebellum, and with Pearson correlation analysis, the relationship between the cerebral and cerebellar lateralization indices.

RESULTS: There was a significant dependency between cerebral and cerebellar language activation, with moderate negative correlation (Pearson $r=-0.69$ ). Crossed cerebrocerebellar language activation was present in both healthy participants and patients, irrespective of handedness or typical or atypical language representation. There were no discordant findings between fMRI and electrocortical stimulation.

CONCLUSIONS: Language lateralization in the cerebellum can be considered an additional diagnostic feature to determine language dominance in patients with brain tumor. This is particularly useful in cases of uncertainty, such as the interference of a brain tumor with cerebral language activation on fMRI and atypical language representation.

ABBREVIATIONS: $\mathrm{ECS}=$ electrocortical stimulation; $\mathrm{HP}=$ healthy participant; $\mathrm{LI}=$ lateralization index

$F_{t a s i n}$ unctional MR imaging is a feasible diagnostic neuroimaging tool for determining hemispheric language dominance in patients with brain tumor preoperatively. ${ }^{1}$ Nevertheless, it has important limitations when evaluating language lateralization, particularly in patients with brain tumors. ${ }^{1-3}$ Activation patterns must be interpreted with great care when the tumor is in or near the presumed language areas, where tumor tissue or mass effect can lead to false-negative fMRI results. ${ }^{2}$

Received June 21, 2014; accepted after revision August 13.

From the Departments of Radiology (C.M.O., M.V., S.K., A.v.d.L., M.S.), Neurology (C.M.O., E.V.-B), and Neurosurgery (E.V.-B., D.S., A.V.), Erasmus MC, University Medical Center Rotterdam, Rotterdam, the Netherlands.

Please address correspondence to Marion Smits, MD, PhD, Department of Radiology (Hs-224), Erasmus MC-University Medical Center Rotterdam, PO Box 2040, 3000 CA Rotterdam, the Netherlands; e-mail: marion.smits@erasmusmc.nl; @MarionSmits

三 Indicates article with supplemental on-line tables.

http://dx.doi.org/10.3174/ajnr.A4147
Determining language dominance is additionally challenging in left-handed patients with brain tumor. Left-handers are known to have less well-defined language-lateralization patterns, with more atypical right-sided language lateralization compared with right-handers. ${ }^{4-6}$ In patients with brain tumor, the ability of fMRI to confirm Wada-established language lateralization was significantly more specific for right- than for left-handed or ambidextrous patients, presumably due to their higher rate of typical, leftlateralized language representation. ${ }^{7}$ In a study evaluating the role of fMRI confirming language dominance in patients with epilepsy, this technique showed higher posttest probability for language dominance in patients with typical language representation than in patients with atypical language representation. ${ }^{8}$

Thus far, studies assessing language lateralization have focused on the supratentorial brain. Prior fMRI studies have shown activation not only in the cerebral hemispheres but also in the cerebellum while performing specific language tasks. ${ }^{9-11}$ An example 
is the verb-generation task, which is preferred to localize language areas in patients with tumor ${ }^{1-14}$ and has been properly validated with electrocortical stimulation (ECS). ${ }^{15}$ In individuals with lefthemispheric language dominance, this task has been shown to activate the right cerebellum. ${ }^{9}$ Some further studies have provided evidence for a so-called crossed cerebrocerebellar language lateralization pattern in healthy individuals, with both typical, left-sided and atypical, right-sided language lateralization. ${ }^{10,16}$ This crossed cerebrocerebellar language lateralization may serve as a useful additional diagnostic feature in determining language hemispheric dominance in patients with brain tumor because the cerebellar language activation is generally undisturbed by the tumor localized in or near the presumed supratentorial language areas. Such an additional diagnostic feature may be especially helpful in patients with potentially atypical language representation, namely left-handers.

The purpose of our study was to assess cerebrocerebellar language fMRI lateralization in healthy participants and in patients with brain tumor, with a focus on atypical language representation.

\section{MATERIALS AND METHODS \\ Study Participants}

We included 20 healthy participants (HPs) whose functional data were reported in a previous study ${ }^{17}$ that investigated the relationship between cerebral functional language lateralization and structural asymmetry of the arcuate fasciculus and 38 patients with brain tumor. Institutional review board approval was obtained for the inclusion of HPs and for the retrospective use of anonymized patient data. We included more left- than righthanded HPs to increase the probability of finding atypical rightlateralized or symmetric language representation. Handedness in healthy participants was assessed with the Oldfield Edinburgh Handedness Inventory. ${ }^{18}$ Written informed consent was obtained from all HPs before participation in this study. The institutional review board waived the requirement to obtain written informed consent from patients. Patients were selected from our data base of 205 patients referred for preoperative fMRI by the neurosurgery department at our institution between May 2004 and September 2013. From this data base, we selected all left-handed patients $(n=19)$ who had performed an fMRI language task. We then matched these with 19 right-handed patients for sex, age, and tumor location. Handedness in all patients was assessed by the neurosurgeon at presurgical neurologic examination. All patients were able to perform the language task and were native Dutch speakers except for 1 native German speaker, who was fluent in Dutch after learning this language at 37 years of age. Nineteen patients (9 left-handed) underwent an operation in an awake setting, where direct ECS was performed to identify language functions (biphasic pulse, $50-\mathrm{Hz}$ frequency, $1-\mathrm{ms}$ duration, 6-12 mA).

\section{Data Acquisition and Preprocessing}

Participants performed a verb-generation task. ${ }^{12}$ They were instructed to think of a verb related to an auditorily presented noun, and in the control condition, they listened to high $(2000-\mathrm{Hz})$ and low $(400-\mathrm{Hz})$ tones.
Participants were scanned at $1.5 \mathrm{~T}$ or $3 \mathrm{~T}$ with an 8 -channel head coil. Functional and structural sequences are specified in On-line Table 1. Imaging data were analyzed by using SPM8 software (http://www.fil.ion.ucl.ac.uk/spm/software/spm8). Functional images were manually aligned to the anterior commissure, realigned to correct for motion, coregistered with the individual's T1-weighed image, ${ }^{19}$ and smoothed with a $3 \mathrm{D}$ Gaussian full width at half maximum filter of $6 \times 6 \times 6 \mathrm{~mm}^{3} .^{20}$

Anatomic images of the HPs were segmented and normalized with the functional images to the Montreal Neurological Institute standard brain space by using affine and nonlinear registration. This process resulted in resampled voxel sizes of $3 \times 3 \times 3 \mathrm{~mm}^{3}$ for the functional and $1 \times 1 \times 1 \mathrm{~mm}^{3}$ for the anatomic images. Patient data were not normalized, in line with routine presurgical analysis, but were resliced with preservation of the acquired voxel size.

\section{Data Analysis}

Statistical activation maps were generated with a general linear model, by using a boxcar function convolved with a hemodynamic response function, corrected for temporal autocorrelation and filtered with a high-pass filter of 128-second cutoff. Motion parameters were included as regressors of no interest to reduce potential confounding effects of motion. Individual t-contrast images of language activation (verb-generation $>$ tones) were generated for all participants. Different from the analysis performed with the HPs in our previous study, ${ }^{17}$ individual lateralization indices (LIs) were determined by ROIs known to be involved in language processing: the inferior frontal gyrus, superior temporal and middle temporal gyri, angular and supramarginal gyri, and the cerebellum. Furthermore, in this study, the number of activated voxels within these ROIs was calculated by using a threshold-independent method. ${ }^{21,22}$ The LI was defined as $(L H-R H) /(L H+R H)$, where $L H$ and $R H$ are the number of activated voxels in the left and right hemispheres, respectively. Activation was classified for the cerebrum and cerebellum separately as left-lateralized for LI values between 0.1 and 1.0, rightlateralized for LI values between -0.1 and -1.0 , or symmetric for LI values between or equal to -0.1 and $0.1 .^{21}$

Patients' individual t-contrast images, thresholded individually but at a minimum t-value of 3.2, were assessed qualitatively by a neuroradiologist with 11 years' experience in presurgical fMRI who was blinded to the handedness of patients and ECS findings. Language activation was assessed in the same predetermined language areas as those quantitatively assessed in the HPs. For each region, activation was categorized as left-lateralized, right-lateralized, symmetric, or no activation, on the basis of which an overall assessment of supratentorial language representation was made. Cerebellar activation was not taken into account when determining overall language lateralization.

For both HPs and patients, when functional language activation in the cerebrum was left-lateralized, we defined this activation as typical language representation. When cerebral language activation was right-lateralized or symmetric, we defined it as atypical language representation. Furthermore, when activation patterns were observed in the left cerebrum and right cerebellum, 
Healthy participant demographics and lateralization indices

\begin{tabular}{|c|c|c|c|c|c|c|}
\hline $\begin{array}{l}\text { Age } \\
\text { (yr) }\end{array}$ & Sex & $\begin{array}{c}\text { EHI } \\
\text { Score }^{a}\end{array}$ & $\begin{array}{l}\text { LI } \\
\text { Cerebrum }\end{array}$ & $\begin{array}{c}\text { LI } \\
\text { Cerebellum }\end{array}$ & $\begin{array}{c}\text { Lateralization } \\
\text { Cerebrum }\end{array}$ & $\begin{array}{c}\text { Lateralization } \\
\text { Cerebellum }\end{array}$ \\
\hline 25 & $M$ & -100 & 0.42 & -0.36 & $\mathrm{~L}$ & $\mathrm{R}$ \\
\hline 30 & $M$ & -100 & -0.44 & 0.34 & $\mathrm{R}$ & $\mathrm{L}$ \\
\hline 33 & $\mathrm{~F}$ & -100 & -0.27 & 0.03 & $\mathrm{R}$ & $S$ \\
\hline 27 & $\mathrm{~F}$ & -100 & 0.34 & -0.41 & $\mathrm{~L}$ & $\mathrm{R}$ \\
\hline 28 & $F$ & -100 & 0.02 & -0.43 & $S$ & $\mathrm{R}$ \\
\hline 35 & $\mathrm{~F}$ & -100 & 0.24 & -0.19 & $\mathrm{~L}$ & $\mathrm{R}$ \\
\hline 53 & $M$ & -90 & -0.18 & 0.13 & $\mathrm{R}$ & $\mathrm{L}$ \\
\hline 36 & $\mathrm{~F}$ & -90 & -0.28 & 0.07 & $\mathrm{R}$ & $S$ \\
\hline 30 & $M$ & -80 & 0.13 & -0.22 & $\mathrm{~L}$ & $\mathrm{R}$ \\
\hline 29 & $M$ & -78 & 0.32 & -0.40 & $\mathrm{~L}$ & $\mathrm{R}$ \\
\hline 28 & $F$ & -20 & -0.04 & -0.13 & $S$ & $\mathrm{R}$ \\
\hline 31 & $M$ & -20 & 0.48 & 0.00 & $\mathrm{~L}$ & $S$ \\
\hline 43 & $M$ & 0 & -0.18 & 0.00 & $\mathrm{R}$ & $S$ \\
\hline 30 & $\mathrm{~F}$ & 100 & 0.21 & -0.30 & $\mathrm{~L}$ & $\mathrm{R}$ \\
\hline 34 & $\mathrm{~F}$ & 100 & 0.16 & -0.04 & $\mathrm{~L}$ & $S$ \\
\hline 34 & $M$ & 100 & 0.32 & -0.12 & $\mathrm{~L}$ & $\mathrm{R}$ \\
\hline 34 & $\mathrm{~F}$ & 100 & 0.31 & -0.46 & $\mathrm{~L}$ & $\mathrm{R}$ \\
\hline 29 & $\mathrm{~F}$ & 100 & 0.42 & -0.30 & $\mathrm{~L}$ & $\mathrm{R}$ \\
\hline 32 & $M$ & 100 & 0.33 & -0.09 & $\mathrm{~L}$ & $S$ \\
\hline 28 & $\mathrm{~F}$ & 100 & 0.43 & -0.25 & $\mathrm{~L}$ & $\mathrm{R}$ \\
\hline
\end{tabular}

Note:- L indicates left-sided; R, right-sided; S, symmetric; EHI, Oldfield Edinburgh Handedness Inventory.

${ }^{a}$ Handedness was assessed with the Oldfield Edinburgh Handedness Inventory.

or vice versa, we defined this relationship as crossed cerebrocerebellar language activation. ${ }^{23}$

\section{Statistical Analysis}

Statistical analysis was performed by using SPSS (Version 20; IBM Armonk, New York). Age differences between left- and righthanded participants were examined by using an independentsamples $t$ test. Sex differences between both groups of patients were examined by using the Fisher exact test. To test whether the lateralizations in the cerebrum and in the cerebellum were independent of each other, we performed a McNemar test in HP and patient data. In HPs, the possible relationship between the LIs of the cerebrum and of the cerebellum was investigated with a scatterplot and was tested with Pearson correlation analysis. We used a significance level of $\alpha=.05$ for all analyses.

\section{RESULTS}

\section{Participant Characteristics}

From the $20 \mathrm{HPs}$ included in the study (mean age, 32.8 years; range, $25-54$ years, 9 men), 13 were classified ${ }^{18}$ as left-handed and 7 , as right-handed (Table). There were no significant differences between the left- and right-handed HPs for age $(t[18]=-0.416$, $P=.682)$ or $\operatorname{sex}(P=.374$, Fisher exact test $)$. Patient characteristics are shown in On-line Table 2. There were no significant differences between the left- and right-handed patients for age $(t[36]=-0.723, P=.474)$ or $\operatorname{sex}(P=.728$, Fisher exact test $)$. In 24 patients, tumors were reported as low-grade, and in 13 patients, as high-grade on histopathologic examination. Thirty-two of the 38 patients underwent surgery. ECS was performed in 19 of these, and language regions were identified in 5 .

\section{Cerebrocerebellar Language Lateralization in HPs}

The cerebral and cerebellar language lateralizations and LIs in HPs are presented in Fig 1 and the Table. We found a crossed cerebrocerebellar activation in $60 \%(12 / 20)$ of HPs. None of the participants showed language lateralization toward the same hemisphere in both the cerebrum and cerebellum. Of the 13 lefthanded participants, 6 showed typical and 7, atypical language representation: right-lateralized cerebral activation in 5 and symmetric cerebral activation in 2 participants. Crossed cerebrocerebellar language activation was observed in 7 left-handed participants (5/6 with typical and 2/7 with atypical language representation). Of the remaining 6 participants, cerebellar activation was symmetric in 4 and right-lateralized in 2 . All 7 righthanded participants showed typical language representation, of whom 5 showed a crossed cerebrocerebellar language activation. The remaining 2 participants showed symmetric activation in the cerebellum.

There was a significant dependency between the cerebral and cerebellar language lateralization patterns with $\chi^{2}(3, n=20)=$ $8.533, P=.036$. The scatterplot (Fig 2 ) indicated a negative direction of this dependency, with a moderate negative correlation between the LIs of the cerebrum and the cerebellum (Pearson $r(20)=-0.69, P=.001)$.

\section{Cerebrocerebellar Lateralization in Patients with Tumor}

The cerebral and cerebellar language lateralizations in patients are presented in Fig 1 and in On-line Table 2. We found crossed cerebrocerebellar activation in $76 \%(29 / 38)$ of patients. None of the patients showed language lateralization toward the same side in both the cerebrum and cerebellum.

Of the 19 left-handed patients, 13 showed typical, and 6, atypical language representation. Crossed cerebrocerebellar language activation was observed in $12 / 13$ left-handed patients with typical language representation. Of the 6 patients with atypical language representation, cerebral language activation was right-lateralized in 1 and symmetric in 5 . Of these, 1 patient with symmetric cerebral language activation showed right-lateralized activation in the cerebellum (Fig $3 A$ ). His tumor was localized in the presumed language areas in the left hemisphere, which could have reduced language activation, resulting in a potentially false symmetric language-activation pattern. In addition, this patient was a bilingual speaker whose mother tongue was German. Although he was fluent in Dutch, he performed the verb-generation task in his mother tongue while the task was presented in Dutch. It is known that language activation in bilingual patients may be more symmetric, ${ }^{24}$ and his performing the task with the interference of both languages may have contributed to the unexpected language-activation pattern we observed. The other patients showed symmetric ( 3 patients) or no activation ( 1 patient) in the cerebellum.

Of the 19 right-handed patients, 17 showed typical and 2, atypical language representation. Crossed cerebrocerebellar language activation was observed in 16 of the 17 patients with typical language representation. Of the 2 patients with atypical language representation, one showed right-lateralized cerebral activation with crossed cerebrocerebellar language representation (Fig 3B) and the other showed symmetric cerebral and cerebellar language activation.

There was a significant dependency between the lateralization in the cerebrum and in the cerebellum $\left[\chi^{2}(6, n=38)=42.06\right.$, $P=.000]$.

There were no discordant findings between fMRI and ECS, which positively identified language representation in the same 


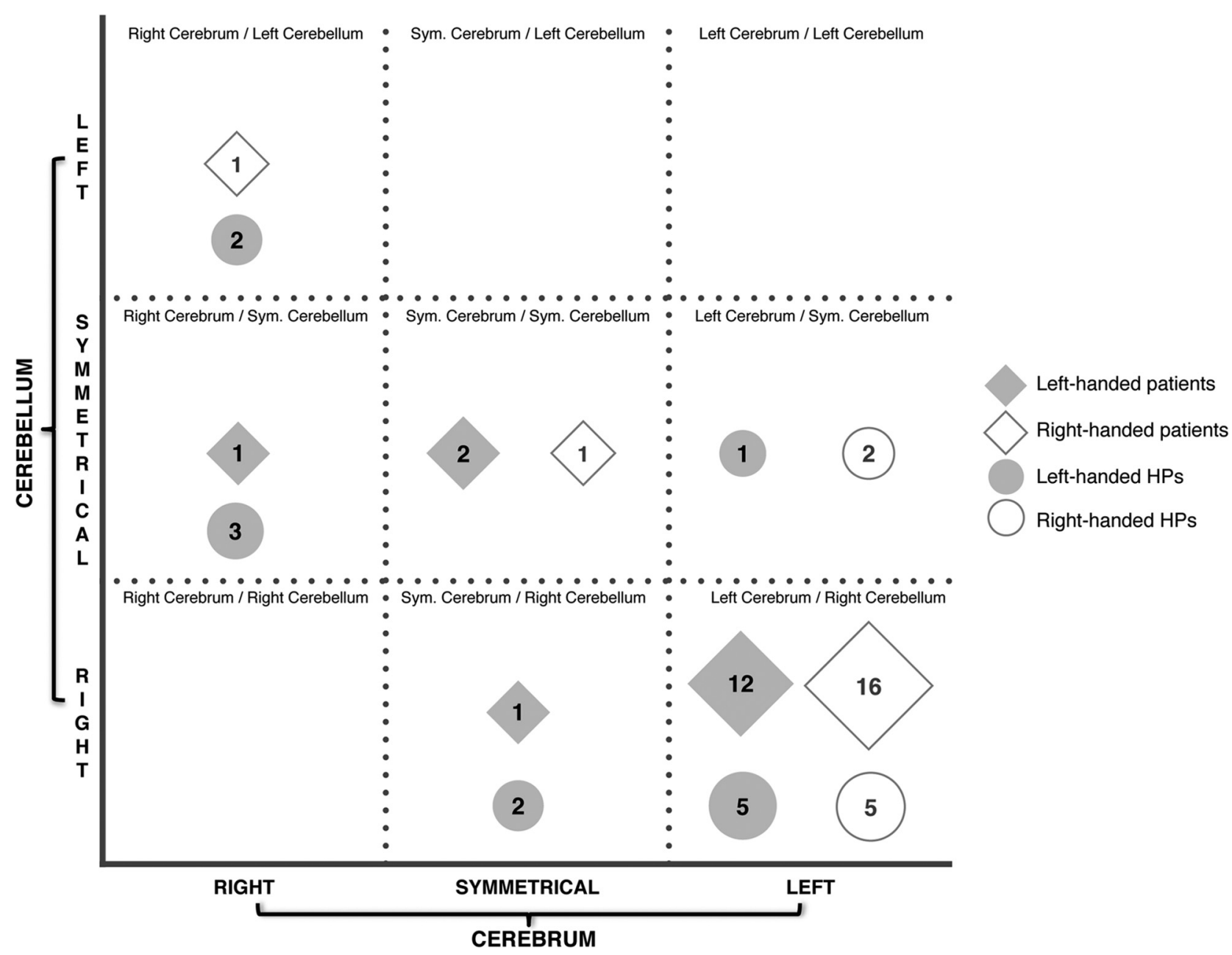

FIG 1. Cerebrocerebellar language representation in healthy participants and patients. The 4 patients with no activation in the cerebellum (as described in On-line Table 2) are not represented in this figure. Sym. indicates symmetric.

hemisphere in 5 patients. In one of these patients, fMRI language activation was found to be present in both hemispheres, whereas it was only identified on the operated side with ECS (the contralateral hemisphere was not assessed with ECS).

\section{DISCUSSION}

We found a significant dependency between language lateralization in the cerebrum and in the cerebellum, both in HPs and in patients with brain tumor, in line with previous studies in healthy left- and right-handers with typical language representation. ${ }^{10,23}$ Furthermore, we found a moderate inverse correlation of cerebrocerebellar lateralization; in other words, the more strongly language was lateralized toward a cerebral hemisphere, the more strongly it was lateralized to the contralateral cerebellar hemisphere. In almost all cases in which activation in the cerebellum was lateralized, there was a crossed cerebrocerebellar lateralization pattern, irrespective of whether language representation was typical or atypical. This means that as a rule of thumb, in cases of clear cerebellar lateralization, cerebral language lateralization can be assumed contralateral. Language lateralization in the cerebellum may thus serve as an additional diagnostic feature for determining hemispheric language dominance in individuals with either typical or atypical language representation. Cerebellar activation was found to be symmetric in a minority of HPs and patients. In these cases, there was no clear correlation with cerebral language representation; in some, cerebral language was also symmetric, but in others, cerebral activation was clearly lateralized. In cases in which assessment of cerebral lateralization is hindered by tumor effects and cerebellar activation is symmetric, another examination such as the Wada test or ECS is thus still required to determine language dominance. ${ }^{1,8}$

Neuroimaging studies of the verb-generation task indicated that the cerebellum is involved in generating or maintaining articulatory representation, ${ }^{25}$ even though no speech motor output was required. Both lesion and functional neuroimaging studies suggest that the cerebellum contributes to diverse cognitive language components and aspects of language production, ${ }^{25,26}$ and cerebellar activation has been reported not only in productive tasks but also in speech-comprehension tasks. ${ }^{27}$ The mechanism underlying the observed crossed cerebrocerebellar activation remains to be elucidated. Connectome analyses could be used to attempt to find white matter pathways that may be responsible for these findings.

Irrespective of handedness or the side of lateralization, crossed cerebrocerebellar activation was observed in most cases and par- 


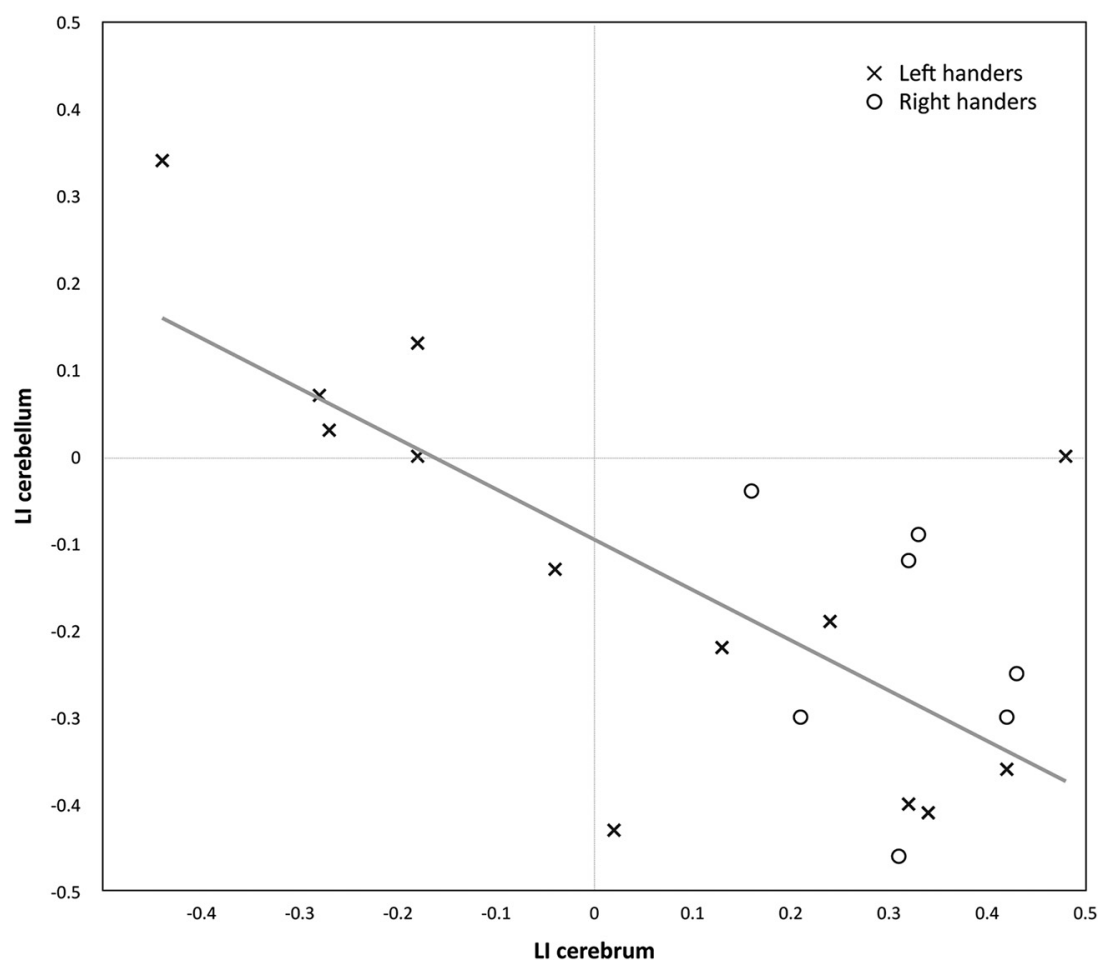

FIG 2. Scatterplot of the healthy participant lateralization indices of the cerebrum and cerebellum.

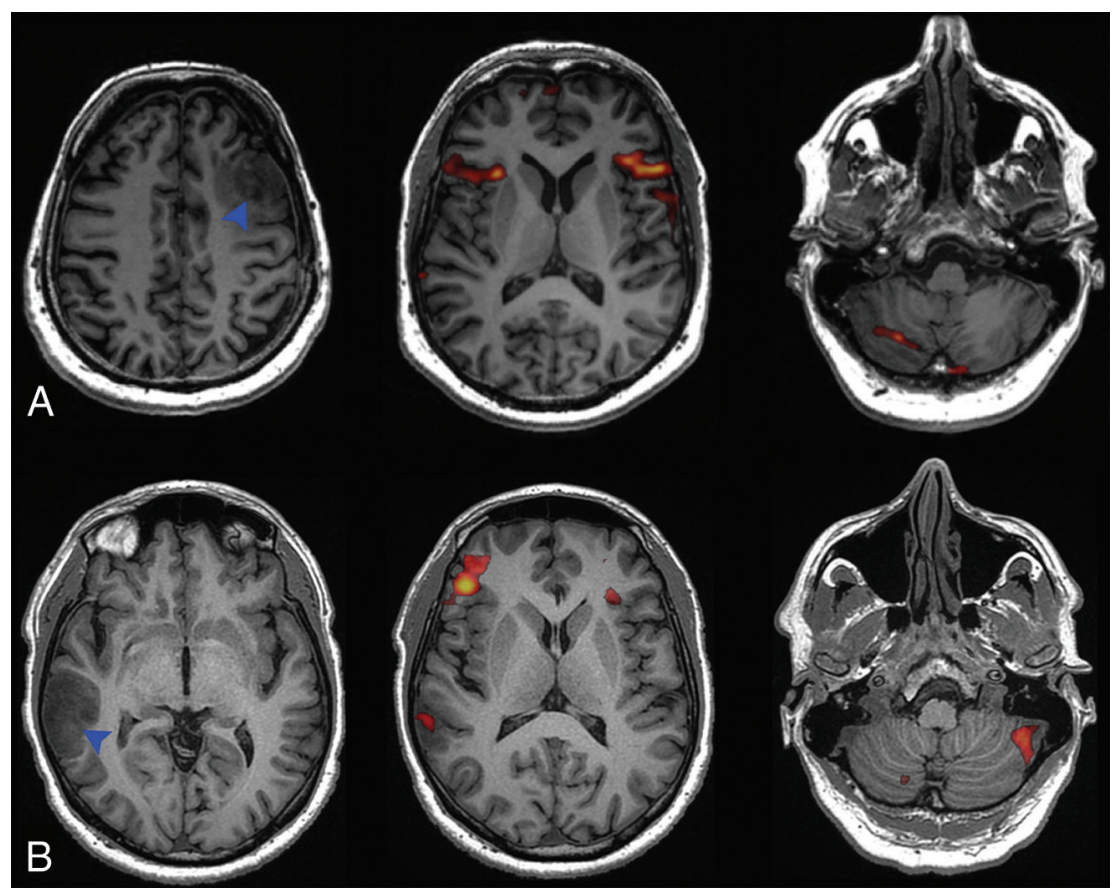

FIG 3. A, Language activation of a left-handed, bilingual patient, with tumor in the left middle frontal gyrus (blue arrowhead), showing symmetric cerebral activation and right-lateralized cerebellar activation. $B$, Language activation of a right-handed patient with tumor in the right middle temporal and angular gyri (blue arrowhead), showing atypical, right-lateralized cerebral activation and crossed cerebellar lateralization.

ticularly in all patients, except one who was bilingual, in whom cerebellar activation was lateralized. This finding is of particular clinical use in cases in which hemispheric language dominance cannot be assessed because of the interference of lan- guage activation due to tumor ${ }^{2,28}$ and in left-handed patients in whom diagnostic uncertainty is greater due to the increased prevalence of atypical language representation.

To the best of our knowledge, this is the first study confirming crossed cerebrocerebellar activation in a large group of HPs and patients with brain tumor with a high prevalence of atypical language representation. To assess the correlation between cerebral and cerebellar lateralization quantitatively, we used a threshold-independent method, which is less prone to within-subject variability than threshold-dependent LI calculation and generates LIs that are more in agreement with clinical findings. ${ }^{21}$ For the assessment of language lateralization in patients with brain tumor, we chose to assess language lateralization qualitatively to remain as close to the clinical routine as possible. Studies comparing quantitative with qualitative assessment of language lateralization have shown that visual inspection by an experienced rater is reliable for presurgical assessment of language lateralization. ${ }^{29-31}$ This qualitative approach provided a clinically applicable assessment of the cerebrocellebellar relationship in patients with tumor.

The main limitation of our study, as in many studies assessing functional language lateralization presurgically, ${ }^{1,32}$ is the relative lack of a criterion standard. Next to techniques such as the Wada test and ECS that are commonly considered criterion standards, transcranial magnetic stimulation, ${ }^{33}$ magentoencephalography, ${ }^{34}$ and fMRI are currently being used as presurgical methods to evaluate language lateralization. In our study, in combination with fMRI, ECS was performed in most of our patients, but in many, no language area was identified. ECS assessment is limited to just the brain region surrounding the tumor, and language areas at some distance from the tumor are thus not identified. Even when a language area is identified, we cannot know for certain whether this is the sole, dominant hemisphere: Bilateral language representation cannot be assessed with certainty. While we found no discordance between ECS and fMRI, the number of patients in whom this could be assessed with certainty was small. A minor limitation of our study is that patients were scanned on several scanners, consistent with 
daily clinical practice. Nevertheless, we used a standardized imaging protocol for presurgical fMRI evaluations, maintaining similar image resolution across our MR imaging systems and standardized image analysis. Another minor limitation was the difference in assessment of handedness: In healthy participants, the standardized Oldfield Edinburgh Handedness Inventory was used while in patients, handedness information was obtained from the preoperative neurosurgical evaluation. Finally, patients with both high- and low-grade gliomas were included in this study. Neurovascular decoupling is a potential limitation of fMRI in high-grade tumors. Our study population was too small to allow a formal comparison or a distinction among these tumor grades.

\section{CONCLUSIONS}

Cerebellar activation may provide an additional diagnostic feature to assess hemispheric language dominance, both in typical and atypical language representations. This is particularly useful in left-handed patients with brain tumor, in whom language representation is commonly atypical, resulting in diagnostic uncertainty, especially when there is potential interference of the tumor with language activation. When cerebellar activation is found to be lateralized, we can, as a rule of thumb, assume that there is contralateral hemispheric language dominance. This crossed cerebrocerebellar pattern of activation could be included as a diagnostic tool in future guidelines of clinical fMRI examinations, which should further specify that a language task that is known to involve the cerebellum is used.

Disclosures: Aad van der Lugt-UNRELATED: Grants/Grants Pending: General Electric Healthcare*; Payment for Lectures (including service on Speakers Bureaus): General Electric Healthcare.* Marion Smits_UNRELATED: Grants/Grants Pending: Erasmus MC, ${ }^{*}$ Coolsingel Foundation*; Payment for Lectures (including service on Speakers Bureaus): European Society for Magnetic Resonance in Medicine and Biology*; Travel/Accommodations/Meeting Expenses Unrelated to Activities Listed: Radiological Society of North America, European Congress of Radiology, European Society for Magnetic Resonance in Medicine and Biology. *Money paid to the institution.

\section{REFERENCES}

1. Stippich C, Rapps N, Dreyhaupt J, et al. Localizing and lateralizing language in patients with brain tumors: feasibility of routine preoperative functional MR imaging in $\mathbf{8 1}$ consecutive patients. Radiology 2007;243:828-36

2. Smits M. Functional magnetic resonance imaging ( $\mathrm{fMRI}$ ) in brain tumour patients. Eur Assoc NeuroOncol Mag 2012;2:123-28

3. Petrella JR, Shah LM, Harris KM, et al. Preoperative functional MR imaging localization of language and motor areas: effect on therapeutic decision making in patients with potentially resectable brain tumors. Radiology 2006;240:793-802

4. Knecht S, Dräger B, Deppe M, et al. Handedness and hemispheric language dominance in healthy humans. Brain 2000;123(pt 12): 2512-18

5. Szaflarski JP, Binder JR, Possing ET, et al. Language lateralization in left-handed and ambidextrous people: fMRI data. Neurology 2002; 59:238-44

6. Tzourio-Mazoyer NN, Josse G, Crivello F, et al. Interindividual variability in the hemispheric organization for speech. Neuroimage 2004;21:422-35

7. Dym RJR, Burns JJ, Freeman KK, et al. Is functional MR imaging assessment of hemispheric language dominance as good as the Wada test? A meta-analysis. Radiology 2011;261:446-55

8. Medina LS, Bernal B, Ruiz J. Role of functional MR in determining language dominance in epilepsy and nonepilepsy populations: a Bayesian analysis. Radiology 2007;242:94-100

9. Binder JR, Frost JA, Hammeke TA, et al. Human brain language areas identified by functional magnetic resonance imaging. $\mathrm{J} \mathrm{Neu}$ rosci 1997;17:353-62

10. Hubrich-Ungureanu PP, Kaemmerer NN, Henn FAF, et al. Lateralized organization of the cerebellum in a silent verbal fluency task: a functional magnetic resonance imaging study in healthy volunteers. Neurosci Lett 2002;319:91-94

11. Frings M, Dimitrova A, Schorn CF, et al. Cerebellar involvement in verb generation: an fMRI study. Neurosci Lett 2006;409:19-23

12. Smits M, Visch-Brink EG, Schraa-Tam CK, et al. Functional MR imaging of language processing: an overview of easy-to-implement paradigms for patient care and clinical research. Radiographics 2006;26:S145-58

13. Wise RJ, Chollet F, Hadar U, et al. Distribution of cortical neural networks involved in word comprehension and word retrieval. Brain 1991;114(pt 4):1803-17

14. Ojemann JG, Ojemann GA, Lettich E. Cortical stimulation mapping of language cortex by using a verb generation task: effects of learning and comparison to mapping based on object naming. J Neurosurg 2002;97:33-38

15. Bizzi A, Blasi V, Falini A, et al. Presurgical functional MR imaging of language and motor functions: validation with intraoperative electrocortical mapping. Radiology 2008;248:579-89

16. FitzGerald DB, Cosgrove GR, Ronner S, et al. Location of language in the cortex: a comparison between functional MR imaging and electrocortical stimulation. AJNR Am J Neuroradiol 1997;18:1529-39

17. Vernooij MW, Smits M, Wielopolski PA, et al. Fiber density asymmetry of the arcuate fasciculus in relation to functional hemispheric language lateralization in both right- and left-handed healthy subjects: a combined fMRI and DTI study. Neuroimage 2007;35:1064-76

18. Oldfield RC. The assessment and analysis of handedness: the Edinburgh inventory. Neuropsychologia 1971;9:97-113

19. Friston KJ, Holmes AP, Poline JB, et al. Analysis of fMRI time-series revisited. Neuroimage 1995;2:45-53

20. Friston KJ, Zarahn E, Josephs $\mathrm{O}$, et al. Stochastic designs in eventrelated fMRI. Neuroimage 1999;10:607-19

21. Suarez RO, Whalen S, Nelson AP, et al. Threshold-independent functional MRI determination of language dominance: a validation study against clinical gold standards. Epilepsy Behav 2009;16: $288-97$

22. Branco DM, Suarez RO, Whalen S, et al. Functional MRI of memory in the hippocampus: laterality indices may be more meaningful if calculated from whole voxel distributions. Neuroimage 2006; 32:592-602

23. Jansen A, Flöel A, Van Randenborgh J, et al. Crossed cerebro-cerebellar language dominance. Hum Brain Mapp 2005;24:165-72

24. Abutalebi J. Neural aspects of second language representation and language control. Acta Psychologica 2008;128:466-78

25. Mariën P, Ackermann H, Adamaszek M, et al. Consensus paper: language and the cerebellum: an ongoing enigma. Cerebellum 2014; $13: 386-410$

26. O'Halloran CJ, Kinsella GJ, Storey E. The cerebellum and neuropsychological functioning: a critical review. J Clin Exp Neuropsychol 2012;34:35-56

27. Londei A, D'Ausilio A, Basso D, et al. Sensory-motor brain network connectivity for speech comprehension. Hum Brain Mapp 2010; 31:567-80

28. Ulmer JL, Hacein-Bey L, Mathews VP, et al. Lesion-induced pseudodominance at functional magnetic resonance imaging: implications for preoperative assessments. Neurosurgery 2004;55:569-79; discussion $580-81$

29. Fernández G, de Greiff A, von Oertzen J, et al. Language mapping in less than 15 minutes: real-time functional MRI during routine clinical investigation. Neuroimage 2001;14:585-94 
30. Jones SE, Mahmoud SY, Gonzalez-Martinez J, et al. Application of a computerized language lateralization index from $\mathrm{fMRI}$ by a group of clinical neuroradiologists. AJNR Am J Neuroradiol 2013;34: $564-69$

31. Gutbrod K, Spring D, Degonda N, et al. Determination of language dominance: Wada test and fMRI compared using a novel sentence task. J Neuroimaging 2012;22:266-74

32. Jones SE, Mahmoud SY, Phillips MD. A practical clinical method to quantify language lateralization in fMRI using whole-brain analysis. Neuroimage 2011;54:2937-49

33. Rösler J, Niraula B, Strack V, et al. Language mapping in healthy volunteers and brain tumor patients with a novel navigated TMS system: evidence of tumor-induced plasticity. Clin Neurophysiol 2014;125:526-36

34. Stufflebeam SM. Clinical magnetoencephalography for neurosurgery. Neurosurg Clin N Am 2011;22:153-67 